\title{
IGF-1-Analogon verbessert Zustand bei Rett-Syndrom
}

\author{
Für Mädchen und Frauen mit Rett-Syndrom gibt es \\ bislang keine spezifische medikamentöse Therapie. \\ Dies könnte sich jedoch bald ändern, sollten sich erste \\ Ergebnisse mit einem IGF-1-Analogon bestätigen.
}

Die Substanz NNZ-2566 ahmt die Funktion des insulinähnlichen Wachstumsfaktors 1 (IGF 1) nach und zeigte in Tierstudien eine Reihe positiver Effekte auf die Gehirnfunktion. Unter anderem ließen sich die synaptische Langzeitpotenzierung und das Dendritenwachstum verstärken, die Wirkung proinflammatorischer Zytokine dämpfen und pathologische Aktivitäten von Astrozyten und Mikroglia unterbinden.

In einer Phase-II-Studie ging es primär um die Sicherheit und Verträglichkeit des IGF-1-Analogons, das unter dem Namen Trofinetide zur Behandlung bei unterschiedlichen Formen von Hirnschädigungen weiterentwickelt werden soll, erläuterte Dr. Daniel Glaze, Universität San Diego/USA. An der Studie nahmen 56 Frauen mit Rett-Syndrom (Alter 16 bis 44 Jahre) teil. Da die Erkrankung im Alter von sechs bis 18 Monaten beginnt und die Gehirnentwicklung in den Folgejahren tiefgreifend beeinträchtigt, war die Erwartung an den Therapieerfolg recht gering.

Neben den Sicherheitsdaten wurden auch Veränderungen im Verhalten der Teilnehmer sowie der Eindruck von Ärzten und pflegenden Angehörigen erfasst. Die stereotypen Handbewegungen von Rett-Patienten wurden mit dem Motor Behavior Assessment (MBA) bewertet, die Schwere des Syndroms auf einer Skala notiert, ebenso die Verbesserung beim klinischen Gesamteindruck (CGI-I). Als Therapieerfolg definiert wurde eine Verbesserung bei mindestens zwei der wichtigsten Parameter, ohne dass es bei anderen zur Verschlechterung kam.

Geprüft wurden 35 sowie 70 mg/kg KG Trofinetide zweimal täglich als Lösung für bis zu vier Wochen. Signifikante Unter- info

Das X-chromosomal vererbte Rett-Syndrom

Etwa eines von 10.000 bis 15.000 Mädchen wird mit einem Defekt im Gen für das Methyl-CpG-Binding Protein 2 (MeCP2) geboren. MeCP2 bindet an methylierte $\mathrm{CpG}$-Inseln und unterdrückt die Transkription verschiedener Gene, unter anderem solche im Cholesterinstoffwechsel. Auch andere Gendefekte können Auslöser sein, zu 95\% ist aber eine Mutation im MeCP2Gen die Ursache. In den ersten Lebensmonaten verläuft die Entwicklung weitgehend normal, dann folgt ein Stillstand und zum Teil Verlust bereits erlernter Fähigkeiten. Sprache und kognitive Fähigkeiten entfalten sich oft nur rudimentär, das Schädelwachstum verzögert sich. Charakteristisch sind stereotype Handbewegungen. Etwa $80 \%$ entwickeln epileptische Anfälle und Beeinträchtigungen, die eine dauerhafte Pflege notwendig machen.

schiede gegenüber Placebo zeigten sich nur in der höheren Dosierung, hier aber in drei der untersuchten Kernparameter: So ging der MBA-Wert mit NNZ-2566 stärker zurück (2,0 versus 0,5 Punkte), und der CGI-I Wert $(3,2$ versus 3,6$)$ deutete auf eine gewisse klinische Verbesserung. Schließlich verringerten sich nach dem Urteil der Pflegepersonen auch die Probleme im Umgang mit den Patienten unter dem Wirkstoff stärker als unter Placebo. Schwere Nebenwirkungen traten unter beiden Dosierungen nicht auf.

NNZ-2566 wird vom US-Unternehmen Neuren entwickelt, das den Wirkstoff nun auch bei Kindern prüfen möchte. Hier besteht die Aussicht, dass die Substanz krankheitsmodifizierend eingreifen und die Ausprägung des Syndroms mildern könnte.

Thomas Müller, Springer Medizin

Oral session 21 - Epilepsy 1. D. Glaze: The Treatment of Rett Syndrome with NNZ-2566. 1. EAN-Kongress Berlin, 20. -23. Juni 2015

\section{Therapierefraktärer Clusterkopfschmerz - jetzt klar definiert}

Drei schwere Anfälle pro Woche trotz Prophylaxe und drei erfolglose Therapieversuche - dann ist nach einem neuen Konsensus-Statement von einem chronischen therapierefraktären Clusterkopfschmerz auszugehen.

Etwa ein Promille der Bevölkerung leidet an Clusterkopfschmerzen, rund ein Sechstel davon an der chronischen Form. Definiert wird diese nach der International Classification of Headache Disorders (ICHD-3 beta) durch Attacken ohne ausreichende Remission oder mit einer Remissionszeit von weniger als einem Monat über ein Jahr hinweg. Diese Definition umfasst jedoch Patienten mit drei Attacken täglich ebenso wie solche mit drei Anfällen monatlich. Sie sagt auch wenig über die Beeinträchtigung der Patienten aus. Der Schweregrad der Anfälle sollte ebenfalls bei der Klassifizierung berücksichtigt werden, so Dr. Dimos Mitsikostas, Präsident der European Headache Federation (EHF).
Ein anderes Problem sieht Mitsikostas bei therapierefraktären chronischen Clusterkopfschmerzen. Wie häufig Patienten mit chronischem Verlauf nicht genügend auf die prophylaktische Behandlung ansprechen, sei auch deshalb nicht klar, weil bisher eine einheitliche Definition von therapierefraktären Schmerzen fehlt. Eine Untersuchung aus dem Jahr 2008 zeigt, dass rund $8 \%$ der Betroffenen auf Prophylaktika nicht ausreichend ansprechen. Damit sei ein therapierefraktärer chronischer Clusterkopfschmerz in der Bevölkerung also eher selten, stellte Mitsikostas fest. Da Clusterkopfschmerzen jedoch mit zu den stärksten Schmerzen überhaupt zählten, sei hier ein effektives Management sehr wichtig, zumal bei unzureichender Wirksamkeit oft nur noch neuromodulatorische und invasive Verfahren übrig blieben, für die es bislang kaum Evidenz gebe. Vor diesem Hintergrund hat die EHF nun einen Expertenkonsensus (www.thejournalofheadacheandpain.com/content/15/1/ 79) erarbeitet. Daran beteiligten sich neben einem EHF-Komi- 